\title{
Reflets
}

Revue ontaroise d'intervention sociale et communautaire

\section{Le Réseau de professionnels à distance : un modèle de prestation de services}

\section{Noël Thomas}

Volume 5, numéro 2, automne 1999

La santé des francophones de l’Ontario

URI : https://id.erudit.org/iderudit/026285ar

DOI : https://doi.org/10.7202/026285ar

Aller au sommaire du numéro

Éditeur(s)

Reflets : Revue ontaroise d'intervention sociale et communautaire

ISSN

1203-4576 (imprimé)

1712-8498 (numérique)

Découvrir la revue

Citer cet article

Thomas, N. (1999). Le Réseau de professionnels à distance : un modèle de prestation de services. Reflets, 5(2), 263-267. https://doi.org/10.7202/026285ar

Tous droits réservés (C) Reflets : Revue ontaroise d'intervention sociale et communautaire, 1999
Ce document est protégé par la loi sur le droit d'auteur. L'utilisation des services d'Érudit (y compris la reproduction) est assujettie à sa politique d'utilisation que vous pouvez consulter en ligne.

https://apropos.erudit.org/fr/usagers/politique-dutilisation/ 


\title{
Le Réseau de professionnels à distance : un modèle de prestation de services
}

\author{
N oël T homas
}

\section{Introduction}

O ffrir une gamme complète de services en français dans une région minoritaire demeure un défi de taille. Les agences sont rarement bilingues, les coûts sont élevés, le financement est inadéquat, les ressources professionnelles ne sont pas disponibles. D ans une approche traditionnelle, on fait venir à grands frais des spécialistes itinérants pour rencontrer occasionnellement la clientèle ou on envoie l'enfant et, par conséquent, sa famille rencontrer un spécialiste dans un grand centre urbain.

Le $R$ egroupement des intervenantes et intervenants francophones en santé et en services sociaux (R IFSSSO) a lancé, en mars 1999, une nouvelle initiative visant à améliorer la prestation et la coordination des services sociaux et de santé en français. Le projet consiste à développer un modèle novateur de prestation en utilisant les technologies de communication.

A ppelé R éseau de professionnels à distance, le modèle veut définir les éléments cliniques, administratifs et financiers ainsi que les indications, contre-indications et conditions de succès pour une initiative de cette nature. 
U ne première étape d'expérimentation clinique en vase clos (à partir de simulations) est prévue pour l'automne 1999, suivie d'une mise en œuvre plus poussée à l'hiver 2000. La région ontarienne de London-W indsor est retenue pour cette première phase, étant donné qu'une entente de collaboration interagence et interministérielle y existe déjà.

\section{Les principes directeurs}

Le projet est guidé par les principes suivants:

- la centration sur la clientèle: le modèle, la gestion et les interventions sont conçues entièrement en fonction d'un bon service à la clientèle;

- un bon service est un service rapide et efficace, sans égard à l'endroit de résidence;

- l'absence de frontières : les professionnels de plusieurs milieux collaborent aux services sans contraintes géographiques, institutionnelles ou ministérielles;

- la responsabilité primaire: on évite de «transférer un cas »à un autre professionnel ou à une autre agence. L'intervenant primaire demeure porteur du dossier pendant la durée de l'intervention;

- le professionnel à distance peut transiger avec le client ou avec le thérapeute primaire ou avec les deux;

- les approches multiples : les services peuvent être offerts en présentiel ou à distance de façon totale ou partielle, selon le besoin;

- les technologies sont utilisées à bon escient, de façon efficace et sans exagération;

- l'accent est mis sur les interactions rapides et utiles: on favorise les interventions intensives et de courte durée. 


\section{Les composantes opérationnelles et les dépendances du modèle}

O n prévoit le cheminement suivant lorsqu'une demande de services est nécessaire. $\mathrm{N}$ otons que chaque étape dépend de la disponibilité de certainesressources, tel qu'indiqué sousla rubrique « dépendance ».

1. Un intervenant de première ligne fait une recherche dans une banque de données sur l'Internet pour un professionnel (dépendance : une banque de données est disponible; l'intervenant de première ligne à accès à l'Internet et sait comment effectuer une recherche; des professionnelsont indiqué leur disponibilité).

2. L'intervenant discute de la situation par téléphone, établit la disponibilité et l'intérêt du professionnel et négocie les détails d'une offre de service. II peut s'agir d'une évaluation, de sessions de consultation auprès de l'intervenant de première ligne, de sessions de traitement du client avec ou sans la participation de l'intervenant de première ligne.

3. L'intervenant et le professionnel s'entendent sur la composante présentielle et la composante médiatisée des interventions (dépendance : les médias sont disponibles et les personnes impliquées veulent et savent les utiliser).

4. Les frais d'intervention sont comptabilisés sur l'Internet de façon dynamique (dépendance: un système de gestion financière interagence est disponible ou possibilité d'un modèle de troc avec ajustements de fin d'année).

\section{Les besoins de formation et de support du modèle}

Pour que le projet atteigne ses objectifs, les personnes impliquées devront apprendre à :

- aider le client via un autre intervenant. C ela pose la question suivante en tant qu'intervenant primaire, comment être le «client » d'un autre professionnel? 
- être consultant ou comment appuyer un intervenant primaire?

- reconnaître leurs forces et leurs limites professionnelles;

- reconnaître les faiblesses et les limites de chaque technologie pour en faire un choix judicieux;

- utiliser les bonnes technologies de la bonne façon.

L'application du modèle dépend aussi d'un encadrement adéquat par les agences, appuyées par les sources subventionnaires.

- La volonté de collaboration interagence et interministérielle doit être documentée dans une entente formelle et doit être communiquée à tout le personnel des agences impliquées.

- Le personnel doit être appuyé par l'employeur et doit être libéré d'une partie de ses tâches traditionnelles pour participer au projet.

- Le projet doit être valorisant pour toutes les personnes impliquées.

- L'agence doit fournir l'équipement nécessaire à l'intervention à distance.

\section{Les questions fondamentales}

Dans le but d'améliorer et finalement de valider le modèle de $R$ éseau de professionnels à distance, on peut se poser les questions suivantes reliées à la prestation de services à distance.

1. Q uelles sont lesindications et lescontre-indications pour une prestation à distance?

2. Q uel équilibre faut-il maintenir entre les activités en présentiel et les activités à distance?

3. Q uelle préparation de la clientèle faut-il prévoir?

4. Q uelle formation faut-il prévoir pour les intervenants de première ligne? Pour les professionnels à distance?

5. Q uelsaménagements cliniques et administratifsfaut-il instaurer? Q uelles politiques et quelles procédures faut-il développer?

6. Q uels encouragements faut-il prévoir pour la clientèle? Pour les intervenants? Pour les professionnels à distance? Pour les agences? 
7. Q uelle formation initiale et continue faut-il planifier? Q uel support technique faut-il offrir aux artisans de la première heure?

8. Quelle boîte d'outils faut-il rendre disponible? Sous quel format?

\section{En conclusion}

Le projet est à la case départ et ce n'est qu'au printemps de l'an 2000 que les premiers résultats seront connus. O n invite la population à participer au cheminement via un site Internet et un babillard à l'adresse électronique suivante : http:/ / village.ca/ sefs/.

Note

Pour des renseignementsadditionnels, on peut aussi communiquer avec $C$ laudia Lebeuf, directrice générale du R IFSSSO par courriel à rifsssoinc@ sympatico.ca ou par téléphone au (416) 9686759.0 n peut rejoindre le responsable du projet, $N$ oëlT homas, à l'adresse électronique suivante : noel.thomas@village.ca ou par téléphone au (613) 748-6996. 